Article

\title{
Hydrazine-Assisted Formation of Indium Phosphide (InP)-Based Nanowires and Core-Shell Composites
}

\author{
Greta R. Patzke ${ }^{1, *}$, Roman Kontic ${ }^{1}$, Zeinab Shiolashvili ${ }^{2}$, Nino Makhatadze ${ }^{2}$ and \\ David Jishiashvili ${ }^{2, *}$
}

1 Institute of Inorganic Chemistry, University of Zurich, Winterthurerstr. 190, Zurich CH-8057, Switzerland; E-Mail: roman.kontic@aci.uzh.ch

2 Institute of Cybernetics, Georgian Technical University, Euli Str. 5, Tbilisi 0186, Georgia; E-Mails: z_shiolashvili@cybernet.ge (Z.S.);n_makhatadze@cybernet.ge (N.M.)

* Author to whom correspondence should be addressed; E-Mails: greta.patzke@aci.uzh.ch (G.R.P.); d_jishiashvili@gtu.ge (D.J.); Tel.: +41-995-9951-5456.

Received: 9 November 2012; in revised form: 19 December 2012 / Accepted: 20 December 2012 / Published: 27 December 2012

\begin{abstract}
Indium phosphide nanowires (InP NWs) are accessible at $440{ }^{\circ} \mathrm{C}$ from a novel vapor phase deposition approach from crystalline InP sources in hydrazine atmospheres containing $3 \mathrm{~mol} \% \mathrm{H}_{2} \mathrm{O}$. Uniform zinc blende (ZB) InP NWs with diameters around $20 \mathrm{~nm}$ and lengths up to several tens of micrometers are preferably deposited on $\mathrm{Si}$ substrates. InP particle sizes further increase with the deposition temperature. The straightforward protocol was extended on the one-step formation of new core-shell InP-Ga NWs from mixed InP/Ga source materials. Composite nanocables with diameters below $20 \mathrm{~nm}$ and shells of amorphous gallium oxide are obtained at low deposition temperatures around $350{ }^{\circ} \mathrm{C}$. Furthermore, InP/Zn sources afford $\mathrm{InP} \mathrm{NWs}$ with amorphous $\mathrm{Zn} / \mathrm{P} / \mathrm{O}$-coatings at slightly higher temperatures $\left(400{ }^{\circ} \mathrm{C}\right)$ from analogous setups. At $450{ }^{\circ} \mathrm{C}$, the smooth outer layer of InP-Zn NWs is transformed into bead-shaped coatings. The novel combinations of the key semiconductor InP with isotropic insulator shell materials open up interesting application perspectives in nanoelectronics.
\end{abstract}

Keywords: indium phosphide; nanowires; semiconductors; gas phase deposition; hydrazine; composite materials 


\section{Introduction}

Among the important group of III-V semiconductor materials [1], indium phosphide (InP) excels through various outstanding properties and finds application in high efficiency photovoltaic cells, integrated circuits and microwave devices operating in the range of $200 \mathrm{GHz}$, especially for the generation, modulation, detection, transmission and amplification of 1.3 and $1.55 \mu \mathrm{m}$ wavelength optical signals. InP has an intermediate band gap of $1.34 \mathrm{eV}$ between $\mathrm{Si}(1.12 \mathrm{eV})$ and GaAs $(1.43 \mathrm{eV})$, and it combines the highest thermoelectric figure of merit among this compound class together with high electron mobility [2-4]. The versatile application potential of InP in forefront technologies, e.g., optoelectronics, spintronics, thermoelectronics and high-speed low-power electronics, as well as in photocatalysis [5], benefits from the formation of InP-based one-dimensional (1D) nanostructures [6]. Single InP nanowire $\mathrm{p}-\mathrm{n}$ junctions have been identified as promising light emitting diode (LED) materials early on [7]. Recently, for example, periodic two-dimensional (2D) arrays of InP nanowires with embedded InAsP segments were found to be essential for controlling directional light emission in nanowire-based LEDs [8], and self-assembled InP nanowires (NWs) are promising photodetectors [9]. The manifold properties of InP NWs can be further enhanced through doping, e.g., with Zn or main group elements [10], and via core-shell heterostructure formation with other semiconductor materials, such as $\mathrm{ZnS}$ [11] or GaP [12]. The majority of these materials have been obtained from MOVPE (metal organic vapor phase epitaxy) or related chemical vapor deposition (CVD) techniques [13,14] proceeding via metal (preferably $\mathrm{Au}$ ) assisted vapor-liquid-solid (VLS) mechanisms [1,15].

We here present a new hydrazine-assisted and catalyst-free gas phase approach [16] for the formation of InP NWs. High aspect ratio InP NWs are directly obtained from InP precursors, and structure and morphology tuning of InP NWs via the growth parameters is discussed. Furthermore, we demonstrate that the novel synthetic strategy can be extended upon the formation of $\mathrm{Zn}$ - and Ga-containing 1D InP core-shell heterostructures.

Whilst the fabrication of 1D nanowire materials with tuned compositions is a challenge in its own right, pristine InP NW growth already gives rise to a fascinating interplay of zinc blende (ZB) and wurtzite (WZ) phases in combination with a variety of twinning phenomena [17]. Although ZB is the preferred structural motif of InP and most other III-V semiconductors, InP NWs often show ZB/WZ polytypism, leading to a staggered type II band alignment in which the $\mathrm{ZB}$ conduction band is $129 \mathrm{meV}$ lower than that of WZ [18-20]. InP NWs preferably grow perpendicular to the close-packed planes of their respective crystal structures, i.e., in ZB-type InP NWs in [111] and WZ-type InP NWs in [0001] direction [21], which renders alternative growth directions, such as [100] ZB InP nanowires, more difficult to access [22]. The presence of twinning and stacking faults considerably influences the NW properties, as illustrated by photoluminescent blue shifts and unexpectedly large photovoltaic effects through rotational twinning in InP NWs [18,23]. Recently, the room temperature conductivity of InP NWs was furthermore found to depend on the longest ZB segment [18]. InP growth phenomena keep attracting preparative and analytical research interest: Growth patterns of tapered ZB/WZ InP nanowires can be tuned via the SA-MOVPE conditions [17], and a first fully resolved structure of twin planes in InP nanowires was obtained from electron image series reconstruction [24].

Doping processes and heterostructure formation through combination of InP with other III-V elements or compounds are powerful strategies to enhance InP NW applications, as illustrated by 
single quantum dot nanowire LEDs of InP-InAsP [13] or InP/InAs/InP core-multishell nanowires [14] obtained via VLS/MOVPE processes. Along these lines, axial $\mathrm{InP} / \mathrm{InSb}$ heterostructures [25] or $\mathrm{InP}_{1-x} \mathrm{Sb}_{x}$ nanowires [26] were furthermore produced from VLS routes.

Despite such rapid progress on InP composites over the past years, there is still room for exploration, as briefly outlined in the following. Combinations of InP with Ga have been reported for $\mathrm{Ga} / \mathrm{InP}$ NWs [27], and 3D (Ga, In)/GaInP structures were grown on polycrystalline InP substrates [28]. Other reported doping elements for InP comprise $\mathrm{Zn}$ [29], Mn [30] and S [13]. However, core-shell composites of $\mathrm{InP}$ with gallium oxide are virtually unexplored, whilst $\mathrm{Ga}_{2} \mathrm{O}_{3}$ coatings of $\mathrm{GaN}$ [31,32] or $\mathrm{ZnO}$ [33] have been fabricated for photocatalytic, sensor and related applications. Given that amorphous $\mathrm{Ga}_{2} \mathrm{O}_{3}$ with a slight excess of $\mathrm{Ga}$ displays a fascinating metal-insulator transition with high application potential in once-write storage devices [34,35], its combination with InP would open up an interesting new class of materials. To the best of our knowledge, only the combination of amorphous $\mathrm{Ga}_{2} \mathrm{O}_{3}$ with $\mathrm{GaN}$ has been reported to date [36].

Zn P-doping of InP [37] is in the focus of theoretical studies [38,39] and has been applied to tune its photoluminescent properties [40], as well as the spacing of twinning superlattices [41]. Moreover, InGaAs/InP transistor performance benefits from $\mathrm{Zn}$ doping [42,43]. Zn-based shell materials for InP NW heterostructures, however, are far less explored, and their combination with oxidic Zn-coatings has not been reported to the best of our knowledge. Few examples, such as TCO ZnO films deposited on InP substrates, still outline the potential of such materials [44].

In the following, we introduce a hydrazine-based direct vapor-solid route to InP nanowires with different structural and twinning motifs. Next, we illustrate the application of this approach upon the all-in-one growth of InP NWs with Ga- and Zn-containing shells. Morphological varieties of Zn-based core-shell InP heterostructures are investigated in detail, and the influence of additional source elements on the InP deposition process is discussed.

\section{Results and Discussion}

\subsection{Hydrazine-Assisted Gas Phase Formation of InP Nanowires}

\subsubsection{Experimental Parameters}

The pyrolytic decomposition of hydrazine in the presence of 3 mol \% $\mathrm{H}_{2} \mathrm{O}$ leads to a variety of highly reactive molecular species that promote the decomposition of the InP source and of gas phase transport processes towards deposition of InP nanowires. As illustrated in previous studies on the formation of phase pure germanium nitride nanowires from a related hydrazine/water atmosphere [16], semiconductor formation is favorable over the oxide formation pathway. The hydrazine-assisted deposition route establishes an elegant one-step and catalyst-free conversion of bulk InP sources into high aspect ratio InP nanowires (NWs) via vapor-solid mechanisms, as discussed in the following.

Sublimation of InP source material in the hydrazine atmosphere of the evacuated quartz reactor provides phosphorus and indium species for the formation of InP nanowires on glass and Si substrates. Phosphorus species can emerge from two processes: First, the saturation pressure of $\mathrm{P}$ is $\mathrm{ca} .10^{-6} \mathrm{~atm}$ at the average preparation temperature of $615{ }^{\circ} \mathrm{C}$, thus providing sufficient quantities for $\mathrm{NW}$ growth [45]. Furthermore, atomic hydrogen generated via hydrazine decomposition has been reported 
to reduce the InP decomposition temperature by $230{ }^{\circ} \mathrm{C}$, thereby promoting the formation of $\mathrm{P}_{2}, \mathrm{P}_{3}$ and $\mathrm{PH}_{3}$ species [46]. Furthermore, indium is formed through decomposition of InP:

$$
\operatorname{InP}(\mathrm{s}) \rightarrow \operatorname{In}(\mathrm{s})+0.5 \mathrm{P}_{2}(\mathrm{~g})
$$

Indium transfer to the substrate surface is probably mediated through the formation of volatile $\operatorname{In}_{2} \mathrm{O}$ molecules emerging from the small quantities of added $\mathrm{H}_{2} \mathrm{O}$ and In. However, further studies in their own right are required to clarify the complex reaction equilibria involved in InP NW deposition.

Concerning the reaction parameters, substrate material and deposition temperature were found to exert a key influence on the phase purity and morphology of the obtained InP products. Glass substrates led to the formation of a mixture of InP and In with no special morphologies, whilst $\mathrm{Si}$ substrates afford phase pure InP nanowires with zinc blende (ZB) structure (Figure 1). Therefore, the following discussion mainly refers to InP NWs grown on Si substrates.

Figure 1. Representative X-ray diffraction (XRD) pattern and scanning electron microscope (SEM) image (inset) of zinc blende (ZB)-type indium phosphide (InP) wires deposited on a Si substrate at $440{ }^{\circ} \mathrm{C}$ (reference pattern: ICSD 73-1983).

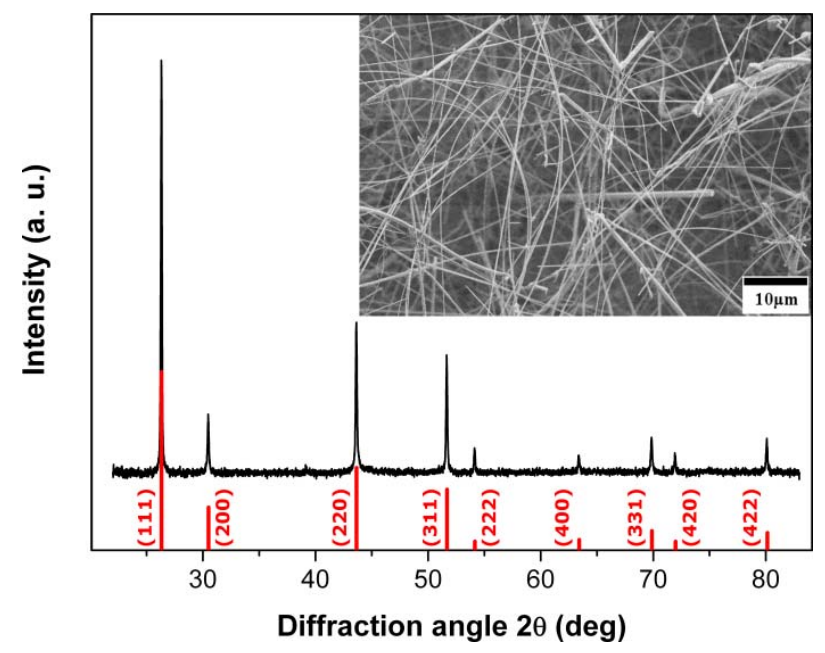

\subsubsection{Temperature-Morphology Relations among InP NWs}

The substrate temperature significantly influences the morphology and crystallinity of the obtained InP materials, which can be differentiated into products obtained at higher temperatures (up to $540{ }^{\circ} \mathrm{C}$ on glass substrates) and NWs grown at $440{ }^{\circ} \mathrm{C}$. Products grown at a substrate temperature of $540{ }^{\circ} \mathrm{C}$ mostly display microscale dimensions with a lower degree of anisotropy and characteristic spherical tips that are indicative of a VLS growth mechanism (Figure S1). High aspect ratio NWs with characteristic zigzag shapes are obtained in smaller quantities and will be discussed further below.

InP NWs grown at $440{ }^{\circ} \mathrm{C}$ on $\mathrm{Si}$ substrates display high aspect ratios with minimum diameters $<40 \mathrm{~nm}$ and lengths $>80 \mu \mathrm{m}$ (inset in Figure 1). The absence of droplet particles at the tips of NWs grown at lower temperatures supports a genuine vapor-solid (VS) growth mechanism. If the same mechanism would be at work at higher and lower temperatures, residual droplets from quenching processes would have been observed after InP deposition at $440{ }^{\circ} \mathrm{C}$ as well. However, their presence at $540{ }^{\circ} \mathrm{C}$ indicates a VLS mechanism (Figure S1), which is changed into VS growth at $440{ }^{\circ} \mathrm{C}$ without 
droplet formation. Furthermore, the absence of tapering (inset in Figure 1) excludes side-wall diffusion or deposition processes for NWs grown at $440{ }^{\circ} \mathrm{C}$ and lower temperatures. TEM investigations of the InP NWs deposited at $440{ }^{\circ} \mathrm{C}$ reveal that they can be differentiated into two NW types (Figure 2). Type 1 NWs (Figure 2a,b) display the characteristic [111] growth direction of ZB-type InP wires, and the observed lattice spacing of $d_{111}=0.340 \mathrm{~nm}$, calculated from the SAED pattern in Figure 2, agrees well with the d-spacing for this plane in ZB InP. Although asymmetric reflections in the SAED patterns indicate a slight structural distortion (Figure 2b), the NWs display uniform diameters, straight walls and little indication of strain or disorder (Figure 2a). Type 2 ZB InP NWs grown along [111] (Figure 2b,c), however, display significant rotational twinning, which can be explained in terms of the low stacking fault energy of the (111) planes in InP [47]. Random twinning is indicated by an irregular sequence of different domains in the TEM image (Figure 2c) and striation of diffraction spots in the SAED (Figure 2d). The stacking order is changed through insertion of twin planes, which create small WZ segments between two ZB domains. The resulting directional change of $39^{\circ}$ leads to the irregularly shaped NW walls. Figure 3 finally shows a third type of ZB InP NWs with a more regular rotational twinning pattern, as shown in preceding reports [48].

Figure 2. Transmission electron microscopy (TEM) images and selected area electron defraction (SAED) patterns of ZB-type InP nanowires grown at $440{ }^{\circ} \mathrm{C}$ (a,b) $92 \mathrm{~nm}$ diameter InP NWs with less defects and corresponding SAED pattern; (c,d) randomly twinned InP NWs with average diameter of $90 \mathrm{~nm}$ and SAED pattern of one of such nanowires, which displays the striation of diffraction spots caused by the introduction of random twins.

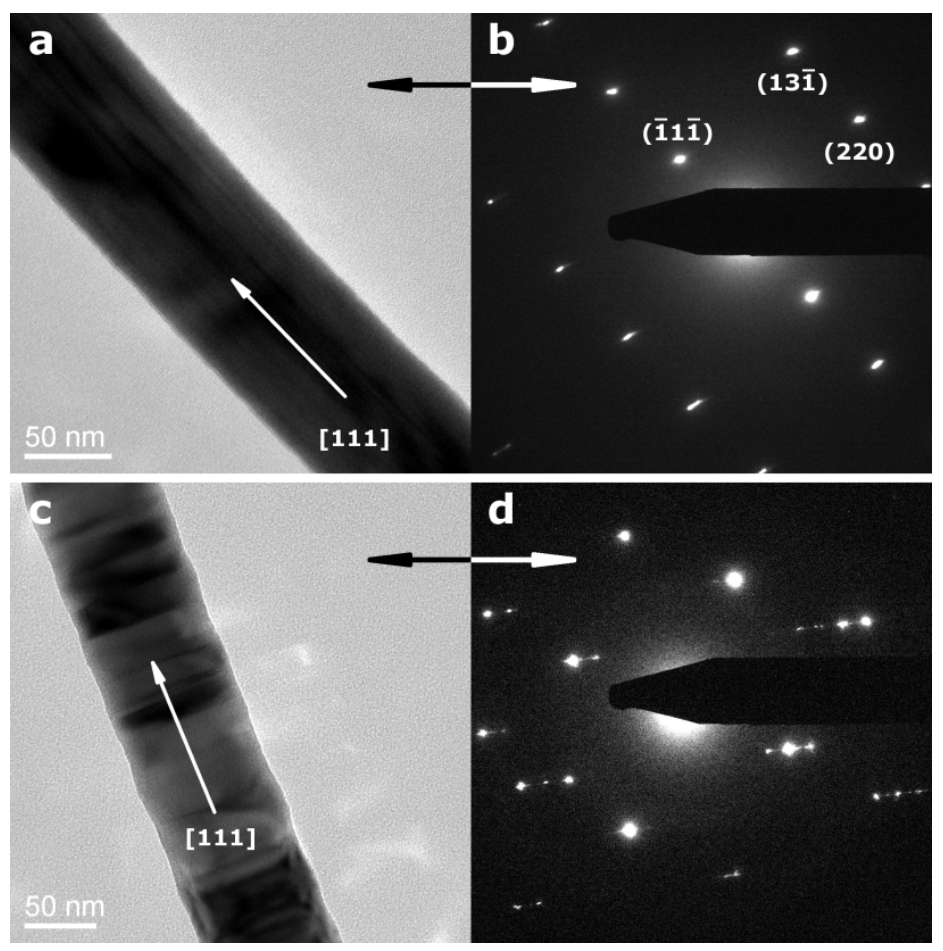

Further structural and elemental analyses on the InP nanowires were conducted with high-angle annular dark field scanning transmission electron microscopy (HAADF-STEM). Z contrast effects clearly indicate that the type $1 \mathrm{InP}$ NWs are indeed uniform in composition and structure, whilst type 2 
NWs display characteristic segmentation due to twinning and WZ segment insertion (Figure S2). EDX analyses on selected spots (Figure S2) are in line with stoichiometric InP. Most importantly, the formation of oxide and nitride side products can be excluded. The deposition temperature of $440{ }^{\circ} \mathrm{C}$ is probably too low to permit InN growth, and oxide side products are likely to be reduced in situ through the generated hydrogen species.

Figure 3. TEM image of more regularly twinned ZB-type InP NWs grown along [111].
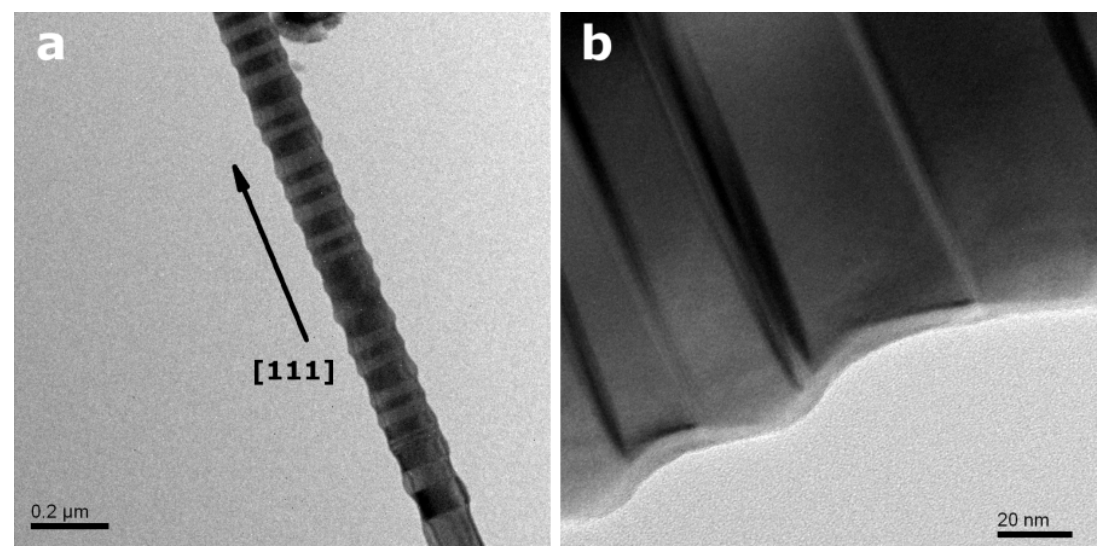

\subsubsection{Zigzag Shaped InP Nanowires}

Figure 4 shows a representative example of ZB-type InP NWs with a characteristic zigzag growth pattern obtained at $540{ }^{\circ} \mathrm{C}$. This morphology attracted considerable interest for device applications due to unique structural and optical properties, e.g., shifts of PL emissions [49,50]. However, preceding routes to such zigzag NWs require the presence of $\mathrm{ZnSe}$ as a promoter and higher formation temperatures of $1200{ }^{\circ} \mathrm{C}$ in quartz tubes. Interestingly, our experimental setup is capable of generating these interesting NW types in the absence of promoters at considerably lower temperatures. Possible driving forces for their formation are induction through hydrazine decomposition products or intense twinning in combination with a high growth rate.

Figure 4. InP nanowire with edged surfaces grown at $540{ }^{\circ} \mathrm{C}$ on a glass substrate (scale bar $=200 \mathrm{~nm})$.

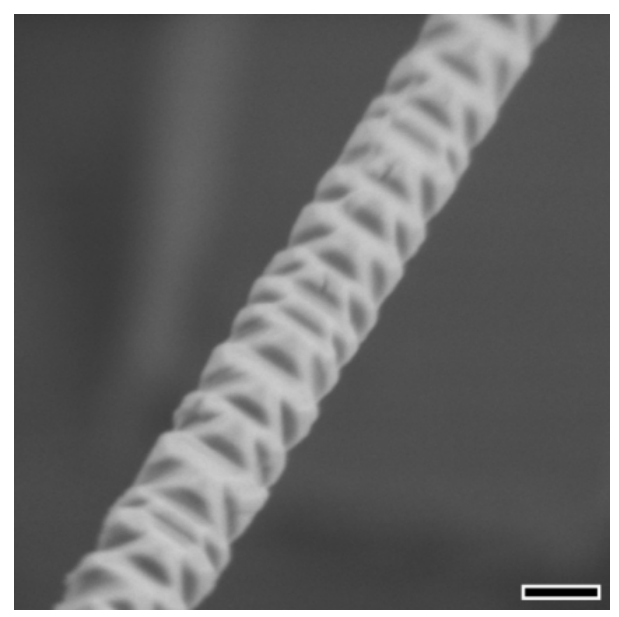




\subsection{Mixed Nanowire Growth from InP-Ga Sources}

\subsubsection{Scope and Synthesis}

As outlined above, the coating of InP nanowires with several types of Ga-containing shells is both of application-oriented and structural research interest. Therefore, the protocol for InP NW growth was modified with respect to the source material. A small piece of pure $\mathrm{Ga}$ was introduced into the reactor together with a $400 \mu \mathrm{m}$ thick single crystalline InP platelet at an approximate InP:Ga surface ratio of 30:1. High aspect ratio NWs are formed from InP-Ga sources in the temperature range between 350 and $425{ }^{\circ} \mathrm{C}$, and neither tapering nor catalyst droplets are observed among the products. Higher deposition temperatures, however, led to less regular wire shapes due to higher growth rates, accompanied by a higher tendency towards stacking faults and twinning. Figure 5 shows a representative PXRD pattern and SEM image of ZB InP-Ga NWs grown at $350{ }^{\circ} \mathrm{C}$ with circular cross sections, lengths up to several tens of micrometers and diameters in the range of 50-100 nm. Other than pristine InP NWs with frequently uneven edges due to rotational twinning, the majority of the InP-Ga NWs display smooth edges, regardless of twinning (Figure S3).

Figure 5. Representative powder X-ray diffraction (PXRD) pattern of ZB InP:Ga nanowires (NWs) $(\mathrm{red}=$ reference pattern: Inorganic Crystal Structure Database (ICSD) 73-1983; green $=\mathrm{Si}$ from substrate) with SEM image of NWs grown at $350{ }^{\circ} \mathrm{C}$ (inset).

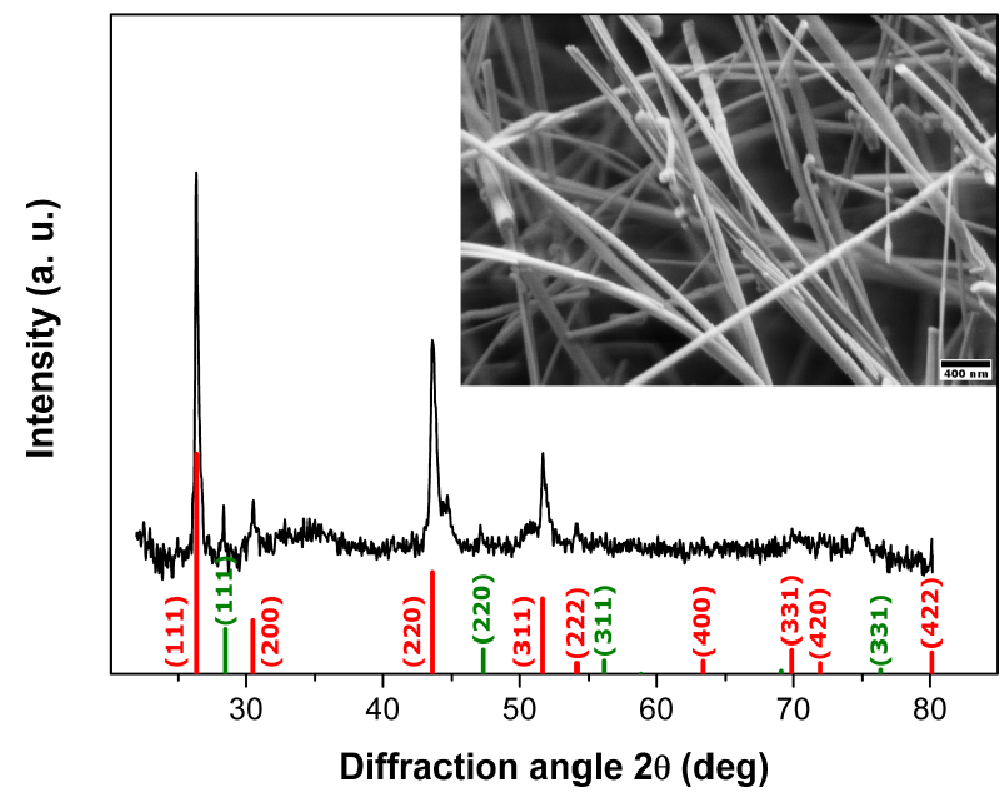

\subsubsection{Structural Properties}

TEM investigations of InP-Ga NWs display a core-shell structure with smooth edges and crystalline cores with diameters smaller than $15 \mathrm{~nm}$, i.e., below the exciton Bohr radius in InP $\left(\mathrm{r}_{\mathrm{B}}=15 \mathrm{~nm}\right)$ [51], thus indicating that the properties of the material are influenced by quantum confinement effects. The addition of $\mathrm{Ga}$ to the source material exclusively promotes the formation of a certain extent of InP-Ga NWs with WZ-type crystalline InP cores [15] (Figure 6c,d) in addition to common ZB-type core (Figure 6a,b) that was obtained from all other setups in the present study. 
Figure 6 illustrates typical growth directions along [111] for the ZB-type and [001] for the WZ-type InP core, respectively.

Figure 6. Representative TEM image of (a) non-twinned ZB-type InP-Ga NW with average core diameter of $14 \mathrm{~nm}$ and average shell thickness of $11 \mathrm{~nm}$; (b) corresponding Fast Fourier Transform (FFT); (c) TEM image of twinned ZB-type InP-Ga NW with average core diameter of $14 \mathrm{~nm}$ and average shell thickness of $8 \mathrm{~nm}$; (d) corresponding FFT.

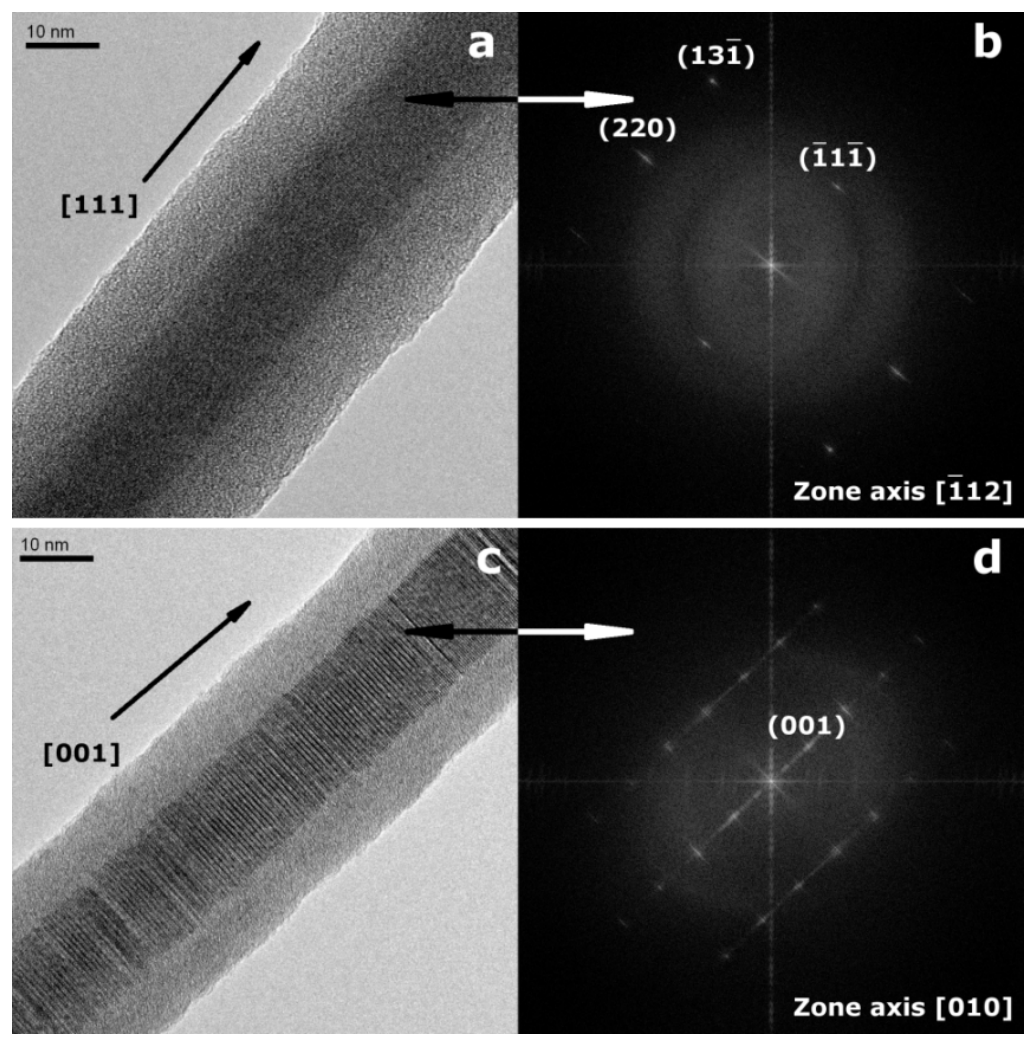

All observed d-spacings for ZB-type InP nanowires fall within the literature data ranges, and they do not point to a lattice parameter decrease, which would be expected in case of a significant Ga incorporation [52]. Interestingly, the NW cores appear to be mostly free of Ga despite its saturation vapor pressure of $\mathrm{ca} .10^{-8}$ Torr at the preparation temperature of $400{ }^{\circ} \mathrm{C}$ in combination with facile sublimation via volatile $\mathrm{Ga}_{2} \mathrm{O}$ molecule formation in the presence of hydrogen and water vapor. These results are backed by HAADF-STEM analyses on NWs with average diameters around $40 \mathrm{~nm}$ in combination with EDX spot measurements (Figure S4). The NW shells consist mainly of Ga and O, whilst the NW cores display massively stronger InP signals and constantly low $\mathrm{Ga} / \mathrm{O}$ ratio. All in all, these results indicate the formation of crystalline InP NWs with an amorphous gallium oxide shell. Given the interesting metal-insulator transition of amorphous $\mathrm{Ga}_{2} \mathrm{O}_{3}$ [34], this new type of NW composites opens up exciting options for theoretical studies. Additionally, the isotropic electrical characteristics of the amorphous $\mathrm{Ga}_{2} \mathrm{O}_{3}$ in the core-shell $\mathrm{InP}-\mathrm{Ga}_{2} \mathrm{O}_{3}$ composite NWs render them interesting candidates for the fabrication of wrap-gate metal insulator semiconductor transistors.

The complex mechanism of subsequent InP and gallium oxide deposition to yield core-shell nanowires is currently under investigation. The absence of Ga doping in the NW core suggests that gallium suboxide molecules are segregated from the growing InP nanowires, followed by their 
oxidation to $\mathrm{Ga}_{2} \mathrm{O}_{3}$ at the nanowire edges. The rather mild formation conditions are probably insufficient to induce recrystallization of gallium oxide, thereby leading to an amorphous gallium oxide coating of the InP nanowires.

\subsection{Hydrazine-Assisted Nanowire Growth from InP-Zn Source Materials}

\subsubsection{Scope and Synthesis}

As $\mathrm{Zn}$-containing shells for InP nanowires are far less explored than bulk doping with $\mathrm{Zn}$ [40,41], the above procedure for the synthesis of $\mathrm{InP}-\mathrm{Ga}$ NWs was modified through replacement of $\mathrm{Ga}$ with $\mathrm{Zn}$ in the source materials. In the following, we demonstrate that the presence of $\mathrm{Zn}$ induces different growth patterns and morphologies of the NW products. Structure and shape of InP-Zn NWs are compared to pristine InP, as well as to InP-Ga NWs to demonstrate the flexibility of the hydrazine-assisted route.

\subsubsection{Influence of the Reaction Temperature}

In analogy to the above-mentioned growth of $\mathrm{InP}$ nanowires, products obtained from $\mathrm{InP}-\mathrm{Zn}$ sources display a tendency towards less regular morphologies at higher temperatures (Figure 7). Uniform and high aspect ratio ZB-type NWs are obtained at $400{ }^{\circ} \mathrm{C}$ (Figures $7 \mathrm{a}$ and 8), and higher deposition temperatures favor the formation of NWs covered with bead-like particles in a rather regular fashion (Figure $7 \mathrm{~b}$ ). The alignment of these bead particles becomes more random with coarsened particle shapes at $650{ }^{\circ} \mathrm{C}$ (Figure $7 \mathrm{c}, \mathrm{d}$ ).

Figure 7. Representative SEM images of nanowires grown from $\mathrm{InP} / \mathrm{Zn}$ sources at (a) $400{ }^{\circ} \mathrm{C}$; (b) $420{ }^{\circ} \mathrm{C}$; (c,d) $650{ }^{\circ} \mathrm{C}$ (scale bars $=2 \mu \mathrm{m}$ for (a-c) and $400 \mathrm{~nm}$ for (d)).
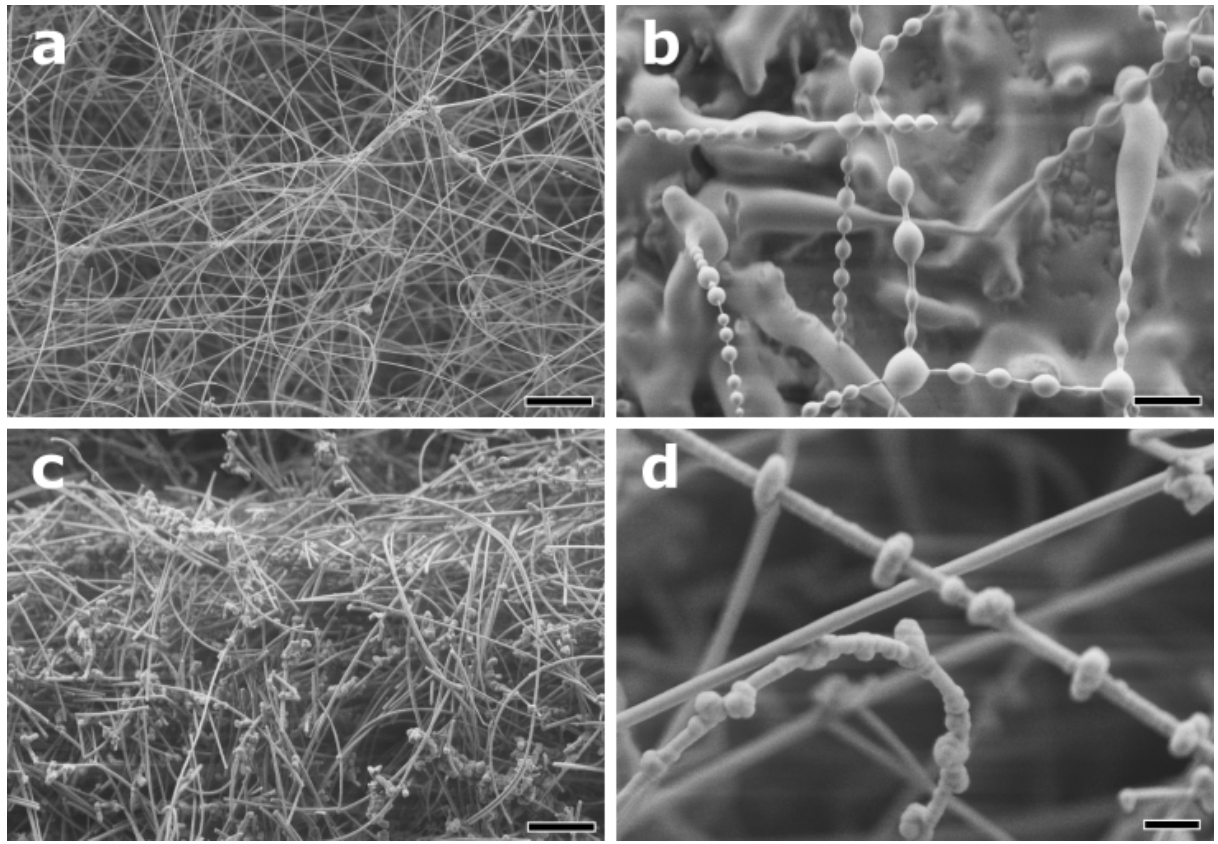
Figure 8. PXRD pattern of high aspect ratio ZB-type NWs grown at $400{ }^{\circ} \mathrm{C}$ from $\mathrm{InP} / \mathrm{Zn}$ sources (reference pattern: ICSD 73-1983).

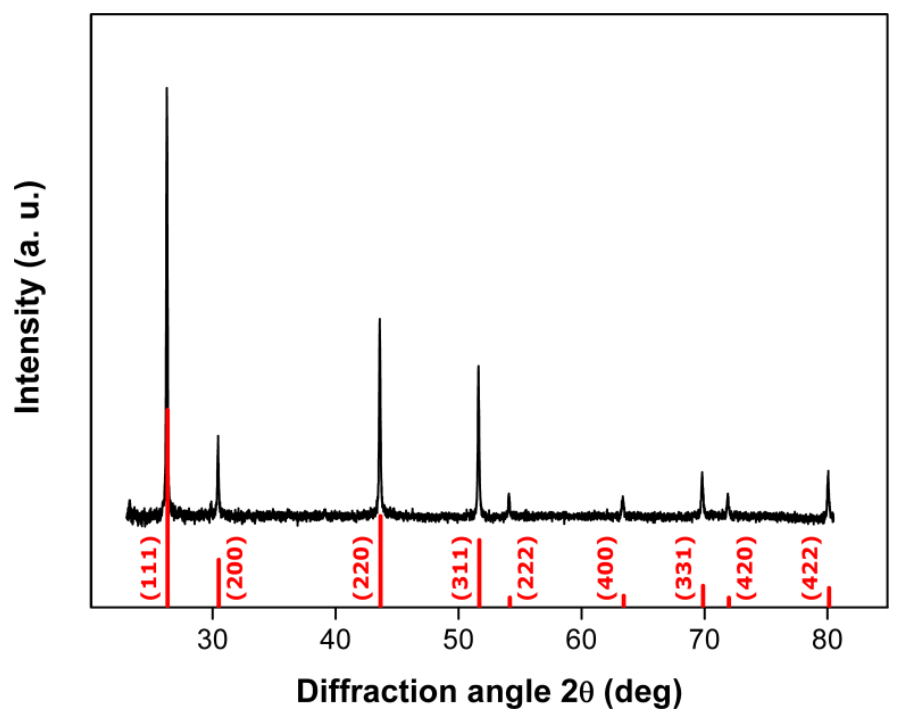

In the following, InP-Zn derived NWs grown at $400{ }^{\circ} \mathrm{C}$ are subjected to a more detailed TEM analysis. Representative TEM images in Figure 9 clearly show the presence of core-shell particles with ZB cores and amorphous shells that bear resemblance to the aforementioned InP-Gallium oxide composites (Figure 6). Interestingly, however, the presence of $\mathrm{Zn}$ in the source material leads to a wider variety of growth directions compared to the pristine $\mathrm{InP}$ and $\mathrm{InP}-\mathrm{Ga}$ systems. Whilst Figure 9a,b shows twinned and untwinned ZB InP rods growing along the expected [111] direction, Figure $9 \mathrm{c}$ illustrates the presence of alternative growth directions, e.g., perpendicular to [111] with the characteristic (111) stacking faults running along the NW axis.

Figure 9. Representative TEM analyses of $\mathrm{ZB}$ nanowires grown from $\mathrm{InP} / \mathrm{Zn}$ sources at $400{ }^{\circ} \mathrm{C}$ : $(\mathbf{a}, \mathbf{b})$ grown along [111]; (c) grown perpendicular to [111] and (d) Fast Fourier Transform of (c).

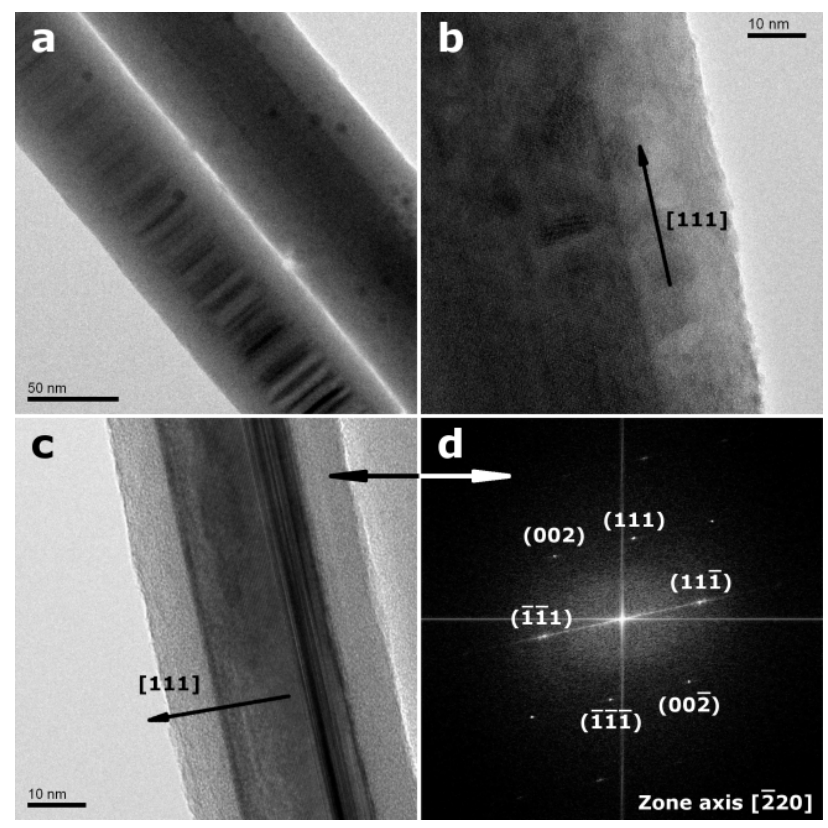


HAADF-STEM analyses coupled with EDX methods (Figure S5) of Zn-containing InP NWs show that $\mathrm{Zn}$ is located in the amorphous NW shell region together with significant amounts of oxygen and phosphorus. Other than in the case of NWs grown from InP-Ga sources, $\mathrm{InP}-\mathrm{Zn}$ based composites are coated with a complex material, probably zinc phosphate. Further investigations on the phase assignment are in progress.

Bead-decorated composite NWs grown at $420^{\circ} \mathrm{C}$ bear close resemblance to InP NWs coated with pearl-shaped $\mathrm{ZnS}$ particles [11]. Whereas the morphologically related $\mathrm{InP}-\mathrm{ZnS}$ NWs were obtained from InP and $\mathrm{ZnS}$ powders at rather high temperature $\left(1250^{\circ} \mathrm{C}\right)$ in preceding studies [11], we lowered the deposition temperature for comparable nano-architectures to $420{ }^{\circ} \mathrm{C}$ with no need for sulfur as an additional precursor element.

The formation of bead-coated InP NWs already sets in to a lower extent at $400{ }^{\circ} \mathrm{C}$, and it is fully developed at $450{ }^{\circ} \mathrm{C}$ (Figure 10). Elevated temperatures might favor shell shrinkage as a result of surface tension, and the resulting local crystallization processes of the NW shell are indicated by the presence of partially crystallized surface beads (Figure 10b). Crystallization at such low temperatures may be enabled by quantum size effects, and elevated temperatures $\left(650{ }^{\circ} \mathrm{C}\right.$, Figure $\left.7 \mathrm{~d}\right)$ are likely to induce Ostwald ripening towards formation of larger surface particles. These complex morphology and crystallinity transformations on the NW surface are subject to follow-up studies.

Figure 10. InP:Zn nanowires covered at $400{ }^{\circ} \mathrm{C}$ with (a) amorphous droplets and (b) crystalline droplets of a $\mathrm{Zn}$-rich shell material.

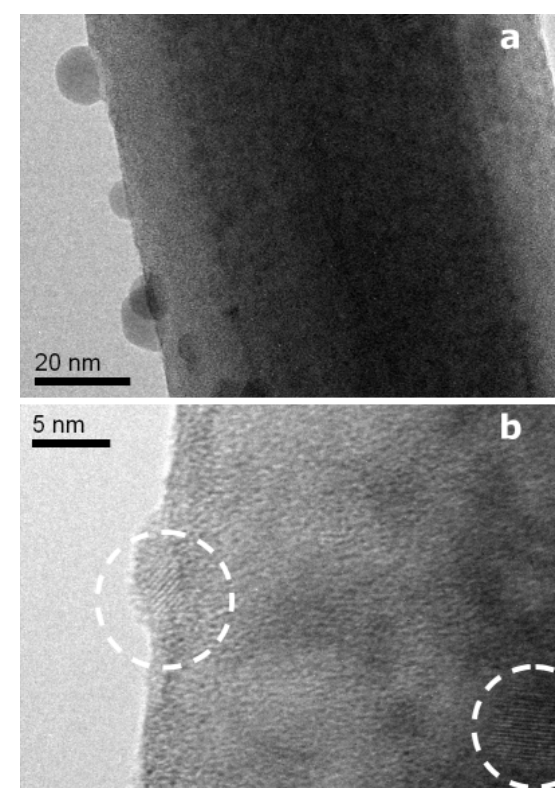

\section{Experimental Section}

\subsection{Sample Preparation}

Commercial grade hydrazine solution in water (50\%, former Soviet standard N5832-65) was distilled at $113{ }^{\circ} \mathrm{C}$ with an Ar flow $(100-150 \mathrm{sccm} / \mathrm{min})$ during the first $20 \mathrm{~min}$. The water content after distillation was determined as 3 mol \% using a IRF-22 refractometer; this remaining water content plays an important role during the synthesis, as discussed below. The reaction was conducted 
in a cylindrical quartz reactor, which was connected to a gas/vacuum system and had a resistive heater attached to its flat bottom.

The source InP wafer (FIET-1 type Te doped (100) oriented single crystalline n-InP with carrier concentration of $5.8 \times 10^{16} \mathrm{sm}^{-3}$ ) was placed at the bottom of the reactor, and the substrate (silicon wafer or glass) was placed above it, separated by a quartz spacer (Figure 11). The reactor was first evacuated and then filled with the saturated pressure of the hydrazine vapor (10 Torr). The temperature of the source was set by the resistive heater. The substrate was subsequently heated due to radiative and convective heating from below, and its temperature was determined by the source temperature and the distance from the source.

Figure 11. Cylindrical quartz reactor for nanowire growth.

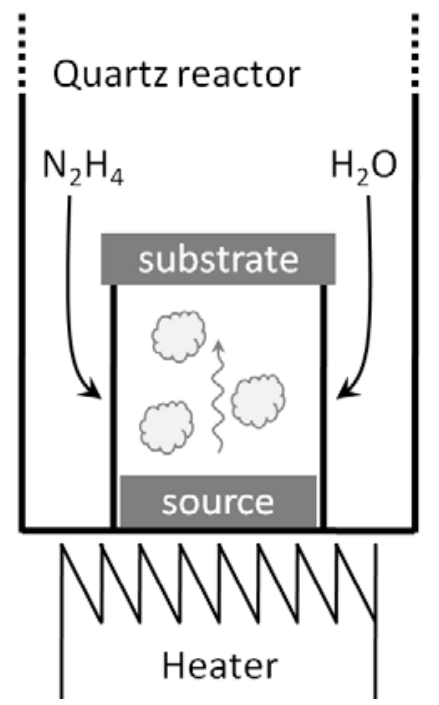

\subsection{Characterization}

Samples were analyzed by scanning electron microscopy (SEM) using a LEO 1530 (FEG) microscope with $1.8 \mathrm{keV}$ electrons. Transmission electron microscopy (TEM) images were recorded on a Philips CM12 operated at $100 \mathrm{keV}$ with a W cathode and a FEI Tecnai F30 (FEG) equipped with an energy dispersive X-ray (EDX) and a high-angle annular dark-field (HAADF) detector; samples were ultrasonicated in ethanol prior to deposition on carbon coated copper grids. X-ray powder diffraction analyses were conducted on a STOE STADI-P2 diffractometer in transmission mode (flat sample holders, Ge monochromated $\mathrm{CuK}_{\alpha 1}$ radiation) equipped with a position-sensitive detector. Samples were fixed with Mylar foil and Wacker P4 silicon glue.

\section{Conclusions}

We established a new one-step vapor-solid approach to high aspect ratio InP NWs via chemical transport of crystalline InP sources in hydrazine $\left(3 \mathrm{~mol} \% \mathrm{H}_{2} \mathrm{O}\right)$ atmospheres. The process affords ZB-type InP NWs with uniform diameters around $20 \mathrm{~nm}$ and lengths up to several tens of nanometers at $440{ }^{\circ} \mathrm{C}$. The clear-cut particle morphologies point to a direct vapor-solid growth mechanism. Reaction temperature is the key parameter for pristine InP nanowire formation, and the particle size increases towards higher deposition temperatures. Furthermore, this flexible preparative route brings 
forward core-shell InP nanowires from mixed precursor materials. InP-Ga sources afford the first example of InP NWs coated with amorphous gallium oxide shells. Nanocables consisting of ZB InP cores with diameters $<20 \mathrm{~nm}$ covered with a thin gallium oxide layer are formed at low deposition temperatures around $350{ }^{\circ} \mathrm{C}$. Likewise, InP-Zn sources generate special core-shell $\mathrm{NW}$ architectures via one-pot and low-temperature hydrazine routes. The process can be steered towards formation of smooth InP NWs with amorphous $\mathrm{Zn} / \mathrm{P} / \mathrm{O}$-shells at $400{ }^{\circ} \mathrm{C}$. They gradually transform into a new type of bead-coated InP:Zn-NWs under mild deposition conditions around $450{ }^{\circ} \mathrm{C}$. These NW composites consist of a crystalline InP core covered by an isotropic amorphous insulator shell, thus opening up new application perspectives in nanoelectronics. Furthermore, the observed morphology transformation processes serve as a starting point for theoretical and crystallographic studies, especially concerning the unprecedented combination of InP with amorphous gallium oxide as a versatile conductor.

\section{Acknowledgments}

This work was supported by the Swiss National Science Foundation (SNSF Professorship PP00P2_133483/1 and SCOPES grant No. IZ73Z0_127943) and financial support from the University of Zurich is gratefully acknowledged. The authors acknowledge support of the Electron Microscopy ETH Zurich, EMEZ, and we thank Frank Krumeich (EMEZ) for TEM analyses.

\section{References}

1. Dick, K.A. A review of nanowire growth promoted by alloys and non-alloying elements with emphasis on Au-assisted III-V Nanowires. Prog. Cryst. Growth Charact. Mater. 2008, 54, $138-173$.

2. Mingo, N. Thermoelectric figure of merit and maximum power factor in III-V semiconductor nanowires. Appl. Phys. Lett. 2004, 84, 2652-2654.

3. Vurgaftman, I.; Meyer, J.R.; Ram-Mohan, L.R. Band parameters for III-V compound semiconductors and their alloys. J. Appl. Phys. 2001, 89, 5815-5875.

4. Riikonen, J.; Tuomi, T.; Lankinen, A.; Sormunen, J.; Säynätjoki, A.; Knuuttila, L.; Lipsanen, H.; McNally, P.J.; O'Reilly, L.; Danilewsky, A.; et al. Synchrotron X-ray topography study of defects in indium antimonide $\mathrm{P}-\mathrm{I}-\mathrm{N}$ structures grown by metal organic vapour phase epitaxy. J. Mater. Sci. Mater. Electron. 2005, 16, 449-453.

5. Chen, H.M.; Chen, C.K.; Lin, C.C.; Liu, R.-S.; Yang, H.; Chang, W.-S.; Chen, K.-H.; Chan, T.-S.; Lee, J.-F.; Tsai, D.P. Multi-bandgap-sensitized $\mathrm{ZnO}$ nanorod photoelectrode arrays for water splitting: An X-ray absorption spectroscopy approach for the electronic evolution under solar illumination. J. Phys. Chem. C 2011, 155, 21971-21980.

6. Agarwal, R. Heterointerfaces in semiconductor nanowires. Small 2008, 4, 1872-1893.

7. Gudiksen, M.S.; Lauhon, L.J.; Wang, J.; Smith, D.C.; Lieber, C.M. Growth of nanowire superlattice structures for nanoscale photonics and electronics. Nature 2002, 415, 617-620.

8. Diedenhofen, S.L.; Janssen, O.T.A.; Hocevar, M.; Pierret, A.; Bakkers, E.P.A.M.; Urbach, H.P.; Gómez Rivas, J. Controlling the directional emission of light by periodic arrays of heterostructured semiconductor nanowires. ACS Nano 2011, 5, 5830-5837. 
9. Pettersson, H.; Zubritskaya, I.; Nghia, N.T.; Wallentin, J.; Borgström, M.T.; Storm, K.; Landin, L.; Wickert, P.; Capasso, F.; Samuelson, L. Electrical and optical properties of InP nanowire ensemble $\mathrm{p}^{+}-\mathrm{i}-\mathrm{n}^{+}$photodetectors. Nanotechnology 2012, 23, 135201-135205.

10. Kubota, E.; Ohmori, Y.; Sugii, K. Electrical and optical properties of Mg-, Ca-, and Zn-doped InP crystals grown by the synthesis, solute diffusion technique. J. Appl. Phys. 1984, 55, 3779-3784.

11. Shen, G.; Chen, P.-C.; Bando, Y.; Golberg, D.; Zhou, Ch. Pearl-like ZnS-decorated InP nanowire heterostructures and their electric behaviors. Chem. Mater. 2008, 20, 6779-6783.

12. Nakaema, M.K.K.; Godoy, M.P.F.; Brasil, M.J.S.P.; Iikawa, F.; Silva, D.; Sacilotti, M.; Decobert, J.; Patriarche, G. Optical and structural investigation of $\operatorname{In}_{1-x} \mathrm{Ga}_{x} \mathrm{P}$ free-standing microrods. J. Appl. Phys. 2005, 98, 053506:1-053506:6.

13. Minot, E.D.; Kelkensberg, F.; van Kouwen, M.; van Dam, J.A.; Kouwenhoven, L.P.; Zwiller, V.; Borgström, M.T.; Wunnicke, O.; Verheijen, M.A.; Bakkers, E.P.A.M. Single quantum dot nanowire LEDs. Nano Lett. 2007, 7, 367-371.

14. Mohan, P.; Motohisa, J.; Fukui, T. Fabrication of InP/InAs/InP core-multishell heterostructure nanowires by selective area metalorganic vapor phase epitaxy. Appl. Phys. Lett. 2006, 88, 133105:1-133105:3.

15. Bhunia, S.; Kawamura, T.; Fujikawa, S.; Nakashima, H.; Furukawa, K.; Torimitsu, K.; Watanabe, Y. Vapor-liquid-solid growth of vertically aligned InP nanowires by metalorganic vapor phase epitaxy. Thin Solid Films 2004, 464-465, 244-247.

16. Jishiashvili, D.; Kapaklis, V.; Devaux, X.; Politis, C.; Kutelia, E.; Makhatadze, N.; Gobronidze, V.; Shiolashvili, Z. Germanium nitride nanowires produced by thermal annealing in hydrazine vapor. Adv. Sci. Lett. 2009, 2, 40-44.

17. Ikejiri, K.; Kitauchi, Y.; Tomioka, K.; Motohisa, J.; Fukui, T. Zinc blende and wurtzite crystal Phase mixing and transition in indium phosphide nanowires. Nano Lett. 2011, 11, 4314-4318.

18. Wallentin, J.; Ek, M.; Wallenberg, L.R.; Samuelson, L.; Borgström, M.T. Electron trapping in InP nanowire FETs with stacking faults. Nano Lett. 2012, 12, 151-155.

19. Muruyama, M.; Nakayama, T. Chemical trend of band offsets at wurtzite/zincblende heterocrystalline semiconductor interfaces. Phys. Rev. B 1994, 49, 4710-4724.

20. Pemasiri, K.; Montazeri, M.; Gass, R.; Smith, L.M.; Jackson, H.E.; Yarrison-Rice, J.; Paiman, S.; Gao, Q.; Tan, H.H.; Jagadish, C.; Zhang, X.; Zou, J. Carrier dynamics and quantum confinement in type II ZB-WZ InP nanowire homostructures. Nano Lett. 2009, 9, 648-654.

21. Kriegner, D.; Wintersberger, E.; Kawaguchi, K.; Wallentin, J.; Borgström, M.T.; Stangl, J. Unit cell parameters of wurtzite InP nanowires determined by X-Ray diffraction. Nanotechnology 2011, 22, 425704:1-425704:7.

22. Wang, J.; Plissard, S.; Hocevar, M.; Vu, T.T.T.; Zehender, T.; Immink, G.G.W.; Verheijen, M.A.; Haverkort, J.; Bakkers, E.P.A.M. Position-controlled [100] InP nanowire arrays. Appl. Phys. Lett. 2012, 100, 053107:1-053107:3.

23. Bao, J.; Bell, D.C.; Capasso, F.; Wagner, J.B.; Mårtensson, T.; Trägårdh, J.; Samuelson, L. Optical properties of rotationally twinned InP nanowire heterostructures. Nano Lett. 2008, 8, 836-841. 
24. Ek, M.; Borgström, M.T.; Karlsson, L.S.; Hetherington, C.J.D.; Wallenberg, L.R. Electron image series reconstruction of twin interfaces in InP superlattice nanowires. Microsc. Microanal. 2011, 17, 752-758.

25. Pozuelo, M.; Zhou, H.; Lin, S.; Lipman, S.A.; Goorsky, M.S.; Hicks, R.F.; Kodambaka, S. Self-catalyzed growth of InP/InSb axial nanowire heterostructures. J. Cryst. Growth 2011, 329, 6-11.

26. Ngo, C.; Zhou, H.; Mecklenburg, M.; Pozuelo, M.; Regan, B.C.; Xiao, Q.F.; Shenoy, V.B.; Hicks, R.F.; Kodambaka, S. Effect of precursor flux on compositional evolution in $\mathrm{InP}_{1-x} \mathrm{Sb}_{x}$ nanowires grown via self-catalyzed vapor-liquid-solid process. J. Cryst. Growth 2011, 336, 14-19.

27. Shen, G.; Bando, Y.; Golberg, D. InP-GaP Bi-coaxial nanowires and amorphous GaP nanotubes. J. Phys. Chem. C 2007, 111, 3665-3668.

28. Sacilotti, M.; Decobert, J.; Sik, H.; Post, G.; Dumas, C.; Viste, P.; Patriarche, G. Structural studies of nano/micrometric semiconducting GaInP wires grown by MOCVD. J. Cryst. Growth 2004, 272, 198-203.

29. van Weert, M.H.M.; Helman, A.; van den Einden, W.; Algra, R.E.; Verheijen, M.A.; Borgström, M.T.; Immink, G.; Kelly, J.J.; Kouwenhoven, L.P.; Bakkers, E.P.A.M. Zinc incorporation via the vapor-liquid-solid mechanism into InP nanowires. J. Am. Chem. Soc. 2009, 131, 4578-4579.

30. Schmidt, T.M.; Venezuela, P.; Arantes, J.T.; Fazzio, A. Electronic and magnetic properties of Mn-doped InP nanowires from first principles. Phys. Rev. B 2006, 73, 235330:1-235330:5.

31. Lee, Y.-K.; Medina, H.; Chiu, P.-W. Modifying optical properties of GaN nanowires by $\mathrm{Ga}_{2} \mathrm{O}_{3}$ overgrowth. J. Vac. Sci. Technol. B 2012, 30, 011802:1-011802:4.

32. Xiao, H.; Pei, H.; Liu, J.; Cui, J.; Jiang, B.; Hou, Q.; Hu, W. Fabrication, characterization, and photocatalysis of $\mathrm{GaN}-\mathrm{Ga}_{2} \mathrm{O}_{3}$ core-shell nanoparticles. Mater. Lett. 2012, 71, 145-147.

33. Jin, C.; Park, S.; Kim, H.; Lee, C. Ultrasensitive multiple networked $\mathrm{Ga}_{2} \mathrm{O}_{3}$-Core/ZnO-shell nanorod gas sensors. Sens. Actuators B 2012, 161, 223-228.

34. Nagarajan, L.; De Souza, R.A.; Samuelis, D.; Valov, I.; Börger, A.; Janek, J.; Becker, K.-D.; Schmidt, P.C.; Martin, M. A chemically driven insulator-metal transition in non-stoichiometric and amorphous gallium oxide. Nat. Mater. 2008, 7, 391-398.

35. Yamaguchi, S. Main group oxides-Making the transition. Nat. Mater. 2008, 7, 353-354.

36. Choi, J.-H.; Ham, M.-H.; Lee, W.; Myoung, J.-M. Fabrication and characterization of $\mathrm{GaN} /$ amorphous $\mathrm{Ga}_{2} \mathrm{O}_{3}$ nanocables through thermal oxidation. Solid State Commun. 2007, 142, 437-440.

37. Hirano, R.; Kanazawa, T.; Inoue, T. Electrical characteristics of $\mathrm{Zn}$ in heavily doped InP grown by the liquid-encapsulated czochralski technique. J. Appl. Phys. 1992, 71, 659-663.

38. Alemany, M.M.G.; Tortajada, L.; Huang, X.; Tiago, M.L.; Gallego, L.J.; Chelikowsky, J.R. Role of dimensionality and quantum confinement in p-type semiconductor indium phosphide quantum dots. Sens. Actuators B 2008, 78, 233101:1-233101:4.

39. Alemany, M.M.G.; Huang, X.; Tiago, M.L.; Gallego, L.J.; Chelikowsky, J.R. The role of quantum confinement in p-type doped indium phosphide nanowires. Nano Lett. 2007, 7, 1878-1882. 
40. van Weert, M.H.M.; Wunnicke, O.; Roest, A.L.; Eijkemans, T.J.; Yu Silov, A.; Haverkort, J.E.M.; 't Hooft, G.W.; Bakkers, E.P.A.M. Large redshift in photoluminescence of p-doped InP nanowires induced by Fermi-level pinning. Appl. Phys. Lett. 2006, 88, 043109:1-043109:4.

41. Algra, R.E.; Verheijen, M.A.; Borgström, M.T.; Feiner, L.-F.; Immink, G.; van Enckevort, W.J.P.; Vlieg, E.; Bakkers, E.P.A.M. Twinning superlattices in indium phosphide nanowires. Nature 2008, 456, 369-372.

42. Duan, Z.; Shi, W.; Chrostowski, L.; Huang, X.; Zhou, N.; Chai, G. Design and epitaxy of $1.5 \mu \mathrm{m}$ InGaAsP-InP MQW material for a transistor laser. Opt. Express 2010, 18, 1501-1509.

43. Ogura, M.; Choi, S.W.; Furue, S.; Hayama, N.; Nishida, K. Effects of Zn doped mesa sidewall on GaIn enhanced InGaAs/InP heterobipolar phototransistor. IEEE J. Quantum Electron. 2010, 46, 214-219.

44. Purica, M.; Budianu, E.; Rusu, E.; Danila, M.; Gavrila, R. Optical and structural investigation of $\mathrm{ZnO}$ thin films prepared by chemical vapor deposition (CVD). Thin Solid Films 2002, 403, 485-488.

45. Ilegems, M.; Panish, M.B.; Arthur, J.R. Phase-equilibria and vapor-pressures in Ga + P system. J. Chem. Thermodyn. 1974, 6, 157-177.

46. Ren, F. III-Nitride based Semiconductor Electronics and Optical Devices and Thirty-Fourth State-of-the-Art Program on Compound Semiconductors (SOTAPOCS XXXIV): Proceedings of the International Symposia; The Electrochemical Society: Pennington, NJ, USA, 2001.

47. Woo, R.L.; Xiao, R.; Kobayashi, Y.; Gao, L.; Goel, N.; Hudait, M.K.; Mallouk, T.E.; Hicks, R.F. Effect of twinning on the photoluminescence and photoelectrochemical properties of indium phosphide nanowires grown on silicon (111). Nano Lett. 2008, 8, 4664-4669.

48. Xiong, Q.; Wang, J.; Eklund, P.C. Coherent twinning phenomena: Towards twinning superlattices in III-V semiconducting nanowires. Nano Lett. 2006, 6, 2736-2742.

49. Shen, G.; Bando, Y.; Liu, B.; Tang, C.; Golberg, D. Unconventional zigzag indium phosphide single-crystalline and twinned nanowires. J. Phys. Chem. B 2006, 110, 20129-20132.

50. Tsuzuki, H.; Cesar, D.F.; de Sousa Dias, M.R.; Castelano, L.K.; Lopez-Richard, V.; Rino, J.P.; Marques, G.E. Tailoring electronic transparency of twin-plane 1D superlattices. ACS Nano 2011, 5, 5519-5525.

51. Byun, H.J.; Lee, J.C.; Yang, H. Solvothermal synthesis of InP quantum dots and their enhanced luminescent efficiency by post-synthetic treatments J. Colloid Interface Sci. 2011, 355, 35-41.

52. Wang, S.Q.; Ye, H.Q. A plane-wave pseudopotential study on III-V zinc-blende and wurtzite semiconductors under pressure. J. Phys. Condens. Matter 2002, 14, 9579-9587.

(C) 2013 by the authors; licensee MDPI, Basel, Switzerland. This article is an open access article distributed under the terms and conditions of the Creative Commons Attribution license (http://creativecommons.org/licenses/by/3.0/). 